\title{
EL PRESUPUESTO POR CENTROS DE ACTIVIDADES EN INSTALACIONES HOTELERAS DEL DESTINO TURISTICO JARDINES DEL REY. UNA NUEVA MIRADA AL PROCESO DE PLANEACIÓN.
}

\section{THE BUDGET BY ACTIVITIES IN DESTINATION HOTEL FACILITIES CENTRES JARDINES DEL REY TOURIST. A NEW LOOK AT THE PLANNING PROCESS.}

Dayana Valdés Pérez, PhD. Doctora en Ciencias Contables y Financieras (Cuba).

Máster en Contabilidad Gerencial. Docente de la carrera de Administración de Empresas en la Pontifica Universidad Católica del Ecuador sede Manabí, Ecuador. dvaldes@pucem.edu.ec

\section{ARTÍCULO DE INVESTIGACIÓN}

Recibido: 15 de agosto de 2016.

Aceptado: 26 de octubre de 2016.

\section{RESUMEN}

El presente artículo es el resultado del estudio realizado en las instalaciones hoteleras del destino turístico Jardines del Rey en la provincia de Ciego de Ávila, Cuba, que tiene como objetivo diseñar un modelo presupuestario por centro de actividad, encaminado a las mejoras de los procesos de planificación y evaluación del desempeño organizacional con una óptica económica. Importantes consideraciones se destacan en el campo teórico enmarcadas en una conceptualización de los principales elementos a considerar en este trabajo, especialmente los conceptos de Presupuesto Basado en Actividades (ABB) y de centro de actividad para instalaciones organizadas en procesos, así como Presupuesto de Ejecución Posterior por Actividad (PEPA), como valiosos instrumentos de gestión. Dentro de los resultados que se enmarcan en el modelo diseñado para este fin, se aprecia la elaboración de un presupuesto para una instalación hotelera muestra, desagregado hasta el nivel de micro actividad, la evaluación económica del desempeño de las estructuras organizativas de la instalación y la concreción del mismo a través de diferentes etapas y pasos metodológicos que facilitan su aplicación, demostrando la importancia de la 
correcta planificación de los recursos para la mejora de la gestión, control y toma de decisiones empresariales.

Palabras clave: Presupuesto por centro de actividad, planificación, centros de actividad, presupuesto de ejecución.

\section{ABSTRACT}

This article is the result of the study conducted in the hotel facilities of the tourist destination Jardines del Rey in the province of Ciego de Avila, Cuba, which aims to develop a model budget by activity centre, aimed at improvements in the processes of planning and evaluation of organizational with a perspective of economic performance. Key considerations stand out in the theoretical field framed in a conceptualization of the main elements to be considered in this work, especially the concepts of budget-based activities (ABB) and activity centre for facilities organized in processes as well as subsequent implementation budget by activity (PEPA), as valuable management tools. Within the results that are framed on the model designed for this purpose, the preparation of a budget for an installation can be seen hotelera displays, disaggregated to the level of micro activity, the economic evaluation of the performance of the installation-organisational structures and the realization through different stages and methodological steps that facilitate its application demonstrating the importance of the correct planning of resources for the improvement of the management, control and business decision-making.

Keywords: Center activity, planning, activity centers and budget, budget execution

\section{INTRODUCCIÓN}

El turismo es considerado mundialmente un sector en constante cambio, sometido con mayor intensidad a los efectos desfavorables de la globalización e internacionalización de los mercados.

Cuba se inserta y lo convierte en un sector priorizado a finales de la década de los ochenta, como una alternativa necesaria para reactivar y desarrollar la economía, de esta forma a inicio de los años noventa tuvo lugar un despegue basado en los excepcionales atractivos de su naturaleza, su rica herencia histórica, cultural y social, su desarrollo sanitario, la estabilidad política que goza y la seguridad para el turista.

Lo anterior condiciona la necesidad de buscar alternativas para que las empresas de este sector se desempeñen eficientemente y sean competitivas. 
En virtud de lo antes mencionado, es incuestionable el empleo de sistemas y mecanismos de planificación, control y manejo de los recursos con un mayor grado de economía, en los que los presupuestos juegan un importante papel, pues contribuyen a una correcta previsión, organización, integración, coordinación y control de las operaciones y resultados de las empresas.

El planteamiento anterior manifiesta la necesidad de elaborar los planes mediante el uso de técnicas que estén a tono con las nuevas tecnologías de gestión de costos, donde los modelos de costo basados en las actividades, convierten en imperativo la necesidad de presupuestar de igual forma, a fin de disponer de una información homogénea para evaluar las desviaciones y el desempeño de los responsables de estas, en cada una de las actividades.

En consecuencia, la presente investigación se refiere específicamente al Presupuesto basado en las actividades, por constituir una potente herramienta de gestión.

Las empresas hoteleras cubanas planifican su economía a través de métodos tradicionales con presupuestos de ingresos y gastos, conformados de manera estática donde la desagregación a las áreas presenta limitaciones por realizarse sobre la base del empirismo, experiencias pasadas, costos históricos o en normas corporativas generales elaboradas a nivel de cadena hotelera. Esto trae, consecuentemente, la notada incapacidad del sistema para adaptarse a los cambios del mundo empresarial caracterizado por la competencia y las innovaciones tecnológicas. Además, bajo estas condiciones, no es posible definir dentro de las actividades del sector cuáles son o no las generadoras de valor para reducir sus costos. Por tanto, la toma de decisiones se ve limitada teniendo en cuenta el patrón de comportamiento al que debe ajustarse la dirección en estos días.

\section{REVISIÓN TEÓRICA}

\section{Consideraciones generales de la presupuestación basada en actividades.}

En el micro entorno de la empresa, es de suma importancia planificar y, al mismo tiempo, retroalimentarse mediante el análisis y control de la misma, pues se obtienen informaciones relevantes para evaluar alternativas y tomar decisiones.

Todas las organizaciones hacen planes. No se puede concebir ninguna, cuyos directivos no estén pensando en definir sus objetivos y métodos para alcanzarlos. Una empresa que no trabaje sujeta a planes, constituye simplemente una masa amorfa incoherente, sin dirección alguna y no propiamente una organización. 
Según (Robles, 2012) la planificación es una técnica de la administración financiera con la cual se pretende el estudio, evaluación y proyección de la vida futura de una organización u empresa, visualizando los resultados de manera anticipada.

Al respecto, se asevera que el costo constituye un indicador de resultado, fundamental punto de partida para realizar reflexiones acerca de la rentabilidad y la eficiencia en cuanto a desempeño organizacional se refiere, razón por la cual su proyección o planificación debe realizarse cuidadosamente.

La planificación financiera según (Gitman, 2012) constituye un aspecto importante de las operaciones de la empresa porque brinda rutas que guían, coordinan y controlan las acciones de la empresa para lograr sus objetivos.

En este contexto juega un papel fundamental el proceso presupuestario, no sólo para prever el futuro sino también para controlar, evaluar la gestión de todos los responsables y tomar las medidas correctoras pertinentes para mejorar el trabajo. El presupuesto es uno de los instrumentos más poderosos de la dirección de la empresa. (Gimenez, 2005) Este es considerado por (Cano, 2013), la expresión cuantitativa de los planes, donde el presupuesto maestro se enfoca en los aspectos operativos, es decir el desarrollo del objeto social y el financiero nos traslada hacia la inversión en activos fijos y a la proyección de estados financieros básicos de propósito general.

Bajo estas concepciones, el mismo se enmarca desde la óptica de su función como categoría económica y herramienta para alcanzar los objetivos empresariales.

Un elemento distintivo que propició el desarrollo presupuestario, lo constituye, el aumento imparable del peso de la actividad pública, exigido por la creciente complejidad e interacción de las economías nacionales e internacionales, que favorece la ruptura del estrecho marco del presupuesto tradicional, de esta forma hace su aparición el Presupuesto basado en las actividades, que es la prolongación de los principios del sistema $A B C$ al área presupuestaria, que se denomina en el lenguaje anglosajón Activity Based Budget (ABB). (Amat O. 2000)

Mediante el $A B B$ se establecen las estimaciones de costos vinculadas a las actividades que deben ser acometidas para producir y vender los productos y servicios; por tanto este, constituye una lógica progresión metodológica a partir del $A B C$.

Al respecto, se puntualiza que la presupuestación basada en las actividades constituye una tecnología de planificación cuyo carácter puede declararse para períodos operativos y/o estratégicos, posibilita elaborar los planes con mayor grado de detalle, parte de los centros de actividad, hasta llegar a las actividades, o segmento organizacional más 
pequeño objeto de plan, cuyos objetivos en una organización estructurada en procesos se corresponden con la meta a alcanzar por estos. En general el ABB, ha devenido en un instrumento de la Contabilidad de Gestión Estratégica.

Por la representación que tienen las actividades en el ABB se considera, una vez realizado un minucioso estudio teórico al respecto, que entre procesos y actividades existe un vínculo estrecho, que provoca en la práctica modos de actuación iguales frente a uno $u$ otro. Por tanto, algunos especialistas empleen indistintamente estos términos. Una actividad en una empresa organizada en procesos, se concreta a partir de la realización de un conjunto de tareas de carácter homogéneo, con una secuencia lógica, que consume recursos, desarrolla un proceso de transformación que genera costos y tiene un output o salida. (Rendón, 2014)

Además, se puede afirmar que están estrechamente vinculadas con el logro de niveles elevados de eficiencia en las empresas.

En la definición abordada anteriormente de Presupuesto basado en actividad, los centros de actividad constituyen el punto de partida, considerándose de suma importancia su análisis de manera que permita su localización, identificación y definición.

Esta terminología es tratada por investigadores que asumen como características esenciales que lo conforman las siguientes:

- Está compuesto por varias actividades con características comunes.

- En las actividades se acumulan costos y gastos.

- Forma parte del sistema contable de la entidad que debe dejar definidas las áreas de responsabilidad correspondientes.

En lo expuesto anteriormente no se incluye la relación de estos con los presupuestos, lo que permitió introducir algunos elementos necesarios en su propuesta investigativa pudiéndolo definir como se muestra a continuación:

Centro de actividad es la agrupación de dos o más actividades que responden a un objetivo y tienen una salida común. Deviene en una estructura que permite elaborar los presupuestos a partir de las salidas con responsables designados que puedan accionar para lograr el cumplimiento de las cifras planificadas. Constituye un segmento de dirección de la empresa, responde y es evaluado en su desempeño por el cumplimiento de su presupuesto de gasto.

Elaborar los presupuestos por centros de actividades posibilita planificar a un nivel intermedio de mando los recursos necesarios para alcanzar las metas y deja sentadas las 
bases para los análisis posteriores, que permitan encauzar cada agrupación al logro de resultados con economía.

Lo anterior permite inferir que la estructura empresarial debe estar soportada por niveles de responsabilidad, estos son identificados con los centros de actividad en el marco de instalaciones empresariales organizadas en procesos.

Las consideraciones anteriores permiten afirmar que los centros de actividad son estructuras organizativas que permiten ser medidas a través de los inductores de costo, por tanto la presupuestación para organizaciones descentralizadas se elabora por centro de actividad, pues esto permite un mayor control de los recursos, si tenemos en cuenta lo planteado por (Robles, 2012) donde define el control financiero como una técnica de la administración que tiene por objeto asegurarse que se lleven a cabo todas las operaciones planteadas inicialmente, de manera eficiente, evaluando de forma conjunta todas las cifras preestablecidas con las reales, para encontrar las desviaciones, deficiencias o fortalezas que ayuden al logro de los objetivos planteados.

La forma más elemental para evaluar el desempeño económico de la organización por centros de actividad, es la comparación a partir de un patrón de referencia. Bajo este concepto el ABB constituye el referente, pero cuando las cifras presupuestadas para los niveles de actividad (producción) previstos no se cumplen, es preciso ajustarlas buscando la homogeneidad de la información para hacerla comparable.

Ante esto, fue valorada la idea de un instrumento que permite solucionar esta problemática: el Presupuesto de Ejecución Posterior por Actividades, que es definido como una técnica que permite el recalculo del ABB cuando el nivel real del inductor no se corresponde con el presupuestado, considera el inductor a nivel unitario y su medida de actividad total, permite realizar análisis a partir de comparaciones y evaluar el desempeño de cada segmento organizativo (centro de actividad) a partir del cumplimiento de las cifras de costos y gastos previstos, posibilita trazar acciones y confeccionar planes de mejora a estos niveles.

Por tanto, se considera que, la evaluación del desempeño para una organización estructurada en centros de actividad, actividad - procesos, tiene como eje cardinal el cumplimiento de las cifras presupuestadas, cuyos directivos serán los responsables de los desempeños más o menos eficientes a ese nivel. Por ello, podrán incidir en este segmento de la estructura organizativa, para reducir las desviaciones en la ejecución real. Una entidad que trabaja con economía se desempeñará eficientemente. 


\section{MATERIALES Y MÉTODOS}

Para cumplir los objetivos propuestos en la investigación se aplicaron métodos teóricos (lógico abstracto, inducción/deducción, análisis y síntesis); así como métodos empíricos tanto para la captación, como para el análisis de la información. Entre ellos se pueden mencionar diferentes técnicas directas e indirectas en la recogida de información. Entre las primeras se destacan: la observación, para determinar las normas de gastos, la revisión documental y el diagnóstico realizado en las instalaciones objeto de pesquisas, las cuales fueron sugeridas por el Ministerio del turismo en Cuba (MINTUR), además, las entrevistas y encuestas: dirigidas a directivos y especialistas de las instalaciones hoteleras, para obtener información sobre el proceso de presupuestación, toma de decisiones y evaluación del desempeño, también a clientes y trabajadores de los hoteles para medir el grado de satisfacción y calidad del servicio.

Las técnicas indirectas se basaron en el análisis de series históricas de los hoteles, que permitieron discernir respecto a los elementos estudiados.

También fueron utilizados métodos estadísticos como: la estadística descriptiva para el procesamiento de las encuestas y modelación econométrica para determinar el tamaño de la muestra intencional (no aleatoria) y proyectar las medidas de actividad total. Todos los análisis se han apoyado en la utilización del paquete profesional SPSS v.12.00 (2003). Para la clasificación y agrupación de las actividades en centros de actividades, se tuvo en cuenta la opinión de 30 especialistas que satisfacen las exigencias de concordancia requeridas por el Método de Criterio de Expertos, valorada según la escala de Liker.

La determinación de las medidas de actividad total y el establecimiento de los coeficientes de gastos fueron predeterminados por actividades, para ello se tuvo en cuenta la magnitud de cada inductor en las diferentes temporadas.

La proyección de estancias se realizó para el año 2013 y se tuvo en cuenta:

Mercado (factor externo).

Capacidad del hotel (factor interno).

Series cronológicas de los años 2000-2012 (tendencia en función de ambos factores para la estimación de la cantidad de turistas días alojados para el año 2013)

\section{ANÁLISIS DE RESULTADOS}

Resultó básica para la clasificación de las actividades de acuerdo a su participación en el servicio o producto la forma en que se vinculan con el mismo. De esta forma, se agruparon en principales y de apoyo (consideradas en los procesos claves y de apoyo). 
Revista Científica ECOCIENCIA

Tabla 1: Clasificación de las actividades y procesos correspondientes.

\begin{tabular}{|l|l|l|}
\hline PROCESOS & ACTIVIDADES PRINCIPALES & ACTIVIDADES DE APOYO \\
\hline Claves & Alimentos & \\
\hline & Bebidas & \\
\hline & Alojamiento y Consejería & \\
\hline & Recreación y animación & Compra de insumos y almacenaje \\
\hline & & Promoción y venta \\
\hline & & Servicios de lavandería \\
\hline & & Servicios de limpieza \\
\hline & & Servicios de comunicaciones \\
\hline & & Servicios técnicos \\
\hline & & Servicios exteriores \\
\hline & & Administración \\
\hline & & Contabilidad y finanzas \\
\hline & & Recursos humanos \\
\hline & & Calidad \\
\hline & & Portadores energéticos \\
\hline
\end{tabular}

Fuente: Elaboración propia a partir de la estructura de procesos definidos por el hotel muestra. 


\section{Identificación de los centros de actividad.}

Tabla 2: Identificación de los centros de actividad y las actividades correspondientes.

\begin{tabular}{|l|l|}
\hline \multicolumn{1}{|c|}{ Centro de Actividad } & \multicolumn{1}{c|}{ Actividad } \\
\hline 1-Gastronomía & $\begin{array}{l}\text { Actividades principales } \\
\text { Alimentos } \\
\text { Bebidas }\end{array}$ \\
\hline 2-Recepción & Servicios de alojamiento y consejería. \\
\hline 3- Recreación y ocio & Recreación y animación \\
\hline \multirow{2}{*}{ 4- Aseguramiento } & $\begin{array}{l}\text { Actividades de apoyo } \\
\text { Compra de insumos } \\
\text { Almacenaje }\end{array}$ \\
\hline 5-Mercadotecnia & Promoción y venta \\
\hline \multirow{2}{*}{ 6-Servicios varios } & $\begin{array}{l}\text { Servicios de lavandería } \\
\text { Servicios de limpieza } \\
\text { Servicios de comunicaciones } \\
\text { Servicios técnicos } \\
\text { S- Infraestructura del hotel }\end{array}$ \\
\hline & $\begin{array}{l}\text { Servicios exteriores } \\
\text { Contabilidad y Finanzas } \\
\text { Recursos Humanos } \\
\text { Calidad } \\
\text { Portadores Energéticos }\end{array}$ \\
\hline
\end{tabular}

Fuente: Elaboración propia 


\section{Determinación de los generadores costos.}

Tabla 3: Detalle de las actividades con su generador de costo.

\begin{tabular}{|l|l|}
\hline Actividades & Generadores de costo \\
\hline Principales & \\
\hline Alojamiento y consejería & Cantidad de estancias \\
\hline Alimentos & Cantidad de estancias \\
\hline Bebidas & Cantidad de estancias \\
\hline Recreación y animación & Cantidad de estancias \\
\hline Apoyo & \\
\hline Compra de insumos & Cantidad de pedidos \\
\hline Almacenaje & Cantidad de pedidos \\
\hline Promoción y venta & Cantidad de estancias \\
\hline Servicios de lavandería & Cantidad de piezas \\
\hline Servicios de limpieza & Metros cuadrados \\
\hline Servicios de comunicaciones & Cantidad de llamadas \\
\hline Servicios técnicos & Órdenes de trabajo \\
\hline Servicios exteriores & Metros cuadrados \\
\hline Administración & Cantidad de estancias \\
\hline Contabilidad y Finanzas & Cantidad de estancias \\
\hline Recursos Humanos & Cantidad de trabajadores \\
\hline Relaciones públicas & Cantidad de estancias \\
\hline Calidad & Cantidad de quejas \\
\hline
\end{tabular}

Fuente: Tomado de tesis doctoral (Rendón, 2014). 


\section{Revista Científica ECOCIENCIA}

Estimación de la medida de actividad total $\left(\boldsymbol{C}_{n}\right)$ y coeficiente de gasto del inductor (In).

Tabla 4: Determinación de las normas para el CA de Gastronomía.

\begin{tabular}{|l|l|l|l|l|l||}
\hline $\begin{array}{l}\text { Centro de } \\
\text { actividad }\end{array}$ & Temporadas & $\begin{array}{l}\text { Norma del } \\
\text { Hotel }\end{array}$ & $\begin{array}{c}\text { Norma de } \\
\text { la cadena }\end{array}$ & $\begin{array}{c}\text { Norma } \\
\text { según } \\
\text { encuestas }\end{array}$ & Promedio \\
\hline Alimentos & Alta & $\$ 8,18$ & $\$ 8,15$ & $\$ 8,20$ & $\$ 8,17$ \\
\cline { 2 - 6 } & Baja & 7,98 & 7,95 & 7,97 & 7,96 \\
\hline Bebidas & Alta & 2,16 & 2,14 & 2,20 & 2,17 \\
\cline { 2 - 6 } & Baja & 1,96 & 1,94 & 2,00 & 1,97 \\
\hline
\end{tabular}

Fuente: Elaboración propia.

Presupuestación de los gastos por centro de actividad.

Tabla 5: Modelo de Presupuestación por centros de actividad $\left(\mathrm{Pca}_{n}\right)$.

\begin{tabular}{|c|c|c|c|}
\hline Centro de actividad/Actividades & 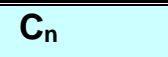 & 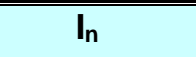 & $\mathrm{Pca}_{\mathrm{n}}$ \\
\hline \multicolumn{4}{|l|}{ Gastronomía } \\
\hline Alimentos & $\$ 8,17$ & 294,277 & $\$ 2,404,243,09$ \\
\hline Bebidas & 2,17 & 294,277 & $638,581,09$ \\
\hline \multicolumn{4}{|l|}{ Recepción } \\
\hline Servicios de alojamiento y consejería & 4,67 & 294,277 & $1,374,273,59$ \\
\hline \multicolumn{4}{|l|}{ Servicios de Animación } \\
\hline Recreación y animación & 0,60 & 294,277 & $176,566,20$ \\
\hline \multicolumn{4}{|l|}{ Aseguramiento } \\
\hline Compra de insumos & 0,69 & 237,007 & $163,534,83$ \\
\hline Almacenaje & 0,60 & 237,007 & $142,204,20$ \\
\hline \multicolumn{4}{|l|}{ Mercadotecnia } \\
\hline Promoción y venta & 0,81 & 294, 277 & $238,364,37$ \\
\hline \multicolumn{4}{|l|}{ Servicios varios } \\
\hline Servicios de lavandería & 1,29 & $1,406,550$ & $1,814,449,50$ \\
\hline Servicios de limpieza & 0,29 & 188,459 & $54,653,11$ \\
\hline Servicios de comunicaciones & 0,40 & 35,966 & $14,386,40$ \\
\hline Servicios técnicos & 0,51 & 4,344 & $2,215,44$ \\
\hline Servicios exteriores & 0,60 & 188,459 & $96,114,09$ \\
\hline \multicolumn{4}{|l|}{ Infraestructura del hotel } \\
\hline Administración & 0,70 & 294,277 & $205,993,90$ \\
\hline
\end{tabular}


Revista Científica ECOCIENCIA

\begin{tabular}{||l|r|r|r||}
\hline \hline Contabilidad y Finanzas & 0,60 & 294,277 & $176,566,20$ \\
\hline Recursos Humanos & 2,89 & 349,00 & $10,080,61$ \\
\hline Calidad & 2,20 & 5,475 & $12,045,00$ \\
\hline Portadores Energéticos & 1,59 & 294,277 & $467,900,43$ \\
\hline Total & & & $\$ 7,992,171,22$ \\
\hline
\end{tabular}

Fuente: Elaboración propia.

Evaluación del desempeño por centros de actividad.

Para evaluar el desempeño se debe ajustar el presupuesto mediante la utilización del PEPA.

El costo real se valoró para 290,500 estancias. A modo de ejemplo, se expone para la temporada alta del año 2013.

Tabla 6: Informe de evaluación del desempeño.

\begin{tabular}{|c|c|c|c|}
\hline Centro de actividad / Actividades & $P p_{n}$ & $\mathrm{Cr}$ & Vr \\
\hline \multicolumn{4}{|l|}{ Gastronomía } \\
\hline Alimentos & $\$ 2,373,385.00$ & $2,765,815.00$ & $(392,430)$ \\
\hline Bebidas & $630,385.00$ & $615,450.34$ & $14,934.66$ \\
\hline \multicolumn{4}{|l|}{ Recepción } \\
\hline Servicios de alojamiento y consejería. & $1,355635.00$ & $1,103,115.62$ & $252,519.38$ \\
\hline \multicolumn{4}{|l|}{ Servicios de Animación } \\
\hline Recreación y animación & $174,300.00$ & $173,150.00$ & $1,150.00$ \\
\hline \multicolumn{4}{|l|}{ Aseguramiento } \\
\hline Compra de insumos & $140,208.00$ & $139,850.00$ & 358.00 \\
\hline Almacenaje & $161,239.20$ & $159,600.00$ & $1,639.20$ \\
\hline \multicolumn{4}{|l|}{ Mercadotecnia } \\
\hline Promoción y venta & $235,305.00$ & $223,262.28$ & $12,042.72$ \\
\hline \multicolumn{4}{|l|}{ Servicios varios } \\
\hline Servicios de lavandería. & $1,810,128.00$ & $1,520,200.00$ & $289,928.00$ \\
\hline Servicios de limpieza & $52,345.00$ & $51,300.00$ & $1,045.00$ \\
\hline Servicios de comunicaciones & $14,784.00$ & $15,780.00$ & 996.00 \\
\hline Servicios técnicos & $2,193.00$ & $2,100.00$ & 93.00 \\
\hline Servicios exteriores & $108,300.00$ & $106,500.00$ & $1,800.00$ \\
\hline
\end{tabular}


Revista Científica ECOCIENCIA

\begin{tabular}{|l|l|l|l||}
\hline Infraestructura del hotel & & & \\
\hline Administración & $203,350.00$ & $202,200.30$ & $1,149.7$ \\
\hline Contabilidad y Finanzas & $174,300.00$ & $173,320.00$ & 980.00 \\
\hline Recursos Humanos & $1,008.61$ & $1,008.61$ & 0 \\
\hline Calidad & $9,790.00$ & $8,850.00$ & 940.00 \\
\hline Portadores Energéticos & $461,895.00$ & $470,500.00$ & $(8,605.00)$ \\
\hline Total & $\$ 7,908,550.81$ & $\$ 7,732,002.15$ & $\$ 176,548.66$ \\
\hline
\end{tabular}

Fuente: Elaboración propia

Como se expuso anteriormente en el año 2013 se aplicó el instrumento diseñado, con el cual se obtuvo el comportamiento que a continuación será objeto de análisis, sin embargo, se consideró oportuno evaluar las variaciones que de forma general mostró la instalación en los años 2011 y 2012, en los que se presupuestó de la forma tradicional.

Tabla 7: Comportamiento del hotel muestra con el presupuesto tradicional. Año 2011.

\begin{tabular}{||c|c|c|c|c||}
\hline Año 2011 & Presupuesto & Real & Variación & \% Cumplimiento \\
\hline \hline Estancias & 367,468 & 356,460 & 11,008 & 2.9 Desfavorable \\
\hline Gasto Total & $\$ 9,934,146.00$ & $\$ 10,281,166.00$ & $\$(347,020,00)$ & 3.5 Sobregasto \\
\hline Por estancia & 27.03 & 28.84 & $(1.81)$ & 6.69 Sobregasto \\
\hline
\end{tabular}

Fuente: Elaboración propia a partir de los datos suministrados por el hotel.

Tabla 8: Comportamiento del hotel muestra con el presupuesto tradicional. Año 2012.

\begin{tabular}{|c|c|c|c|c|}
\hline Año 2012 & Presupuesto & Real & Variación & \% Cumplimiento \\
\hline \hline Estancias & 302,450 & 279,500 & 22,950 & 7.58 Desfavorable \\
\hline Gasto Total & $\$ 9,125,790.00$ & $\$ 9,628,893.00$ & $\$(503,103.00)$ & 5.51 Sobregasto \\
\hline Por estancia & 30.17 & 34.45 & $(4.28)$ & 14.18 Sobregasto \\
\hline
\end{tabular}

Fuente: Elaboración propia a partir de los datos suministrados por el hotel.

Como se aprecia en las tablas anteriores en ambos años se obtuvieron sobregastos de $3,5 \%$ y $5,5 \%$ respectivamente, por estancias estos fueron aún mayores, en el análisis de las causas, a simple vista se observa que se produjo una reducción en la cantidad de estancias, sin embargo, a partir del estudio de los documentos y de la entrevistas con directivos se pudo constatar que en el caso de los centros de actividad principales (en esos años no fue empleado esta forma de agrupación), se proyectó en base a normas 
solo en la actividad de alimentos y bebidas, el alojamiento careció de estas, y de la misma forma sucedió con otras áreas indirectas al servicio, es decir, se presupuestó en base a los contratos previstos de arribos y los gastos se sustentaron básicamente en comportamientos históricos, ello justifica un cumplimiento tan desacertado.

La comparación del presupuesto basado en las actividades con los costos reales de cada una de ellas reflejados en la tabla No. 6 muestra desviaciones favorables (Real menor que el Plan), localizándose sólo movimientos desfavorables en la actividad principal de Alimentos (sobregasto del 1,2\%) y la actividad de apoyo Portadores Energéticos (sobregasto 1,1\%).

Es oportuno destacar que la mejora que se aprecia en el año 2013 no fue solo el efecto de la aplicación del Presupuesto por Centros de Actividades, aunque el seguimiento y control sistemático y con el grado de detalle que se realizó (por centro, por actividad, decenal y mensual) posibilitó ir realizando ajustes para que al cierre del año no sucediera lo que en años anteriores.

Por tanto, podemos afirmar que la interrelación existente entre las etapas que forman parte de este procedimiento manifiesta la factibilidad de la utilización del mismo y su incidencia en la mejora del proceso de planificación económica para instalaciones hoteleras; además, expone la forma en que el instrumento concebido permite evaluar el desempeño organizacional con un enfoque económico.

\section{CONCLUSIONES}

El Modelo de Presupuestación por Centros de Actividad combina de manera adecuada dos instrumentos de gestión que se interrelacionan y posibilitan evaluar el desempeño.

El Presupuesto por Centros de Actividad, aporta el establecimiento de coeficientes para los generadores de costo, que pueden ser tratados como normas, factibles de ser utilizadas, para cualquier nivel de proyección en correspondencia con el inductor, situando a disposición de los directivos una valiosa información.

La desagregación del presupuesto garantiza la planificación con mayor grado de detalle hasta los segmentos más pequeños de la organización.

Las técnicas utilizadas para planear por actividades constituyen una forma superior de proyección, que tienen en cuenta herramientas con mayor grado de rigor, lo cual eleva la calidad del proceso de planificación y la toma de decisiones en instalaciones hoteleras. 
La comparación entre la forma tradicional de elaborar los presupuestos y la utilizada a partir de las etapas y pasos metodológicos, demuestra la superioridad del Modelo de Presupuestación por Centros de Actividad.

El Presupuesto por Centros de Actividad constituye un eficaz instrumento para evaluar el desempeño y un tributo a la educación económica de los directivos y trabajadores de las instalaciones hoteleras.

\section{REFERENCIAS BIBLIOGRÁFICAS}

Amat, J. P. (2002). Control presupuestario. Barcelona españa: 2da edición, Gestión 2000.

Amat, O. y. (2000). Contabilidad y gestión de costes. España: Gestión 2000.

Bravo Valdivieso, M., \& Ubidia, T. C. (2007). Contabilidad de costos (Primera ed.). Quito, Ecuador: NUEVODIA.

Burbano, R. J. (2011). Presupuestos, un enfoque de direccionamiento estratégico, gestión y control de recursos. (Cuarta ed.). Bogotá, Colombia: McGrawHill Educación.

Cano, M. A. (2013). Contabilidad gerencial y presupuestaria. aplicada a las ciencias económicas, administrativas y contables. Bogotá, Colombia: Ediciones de la U.

Gimenez, C. y. (2005). Costos para empresarios. Mexico: Macchi.

Gitman, J. y. (2012). Principios de administración finaciera (Decimosegunda ed.). Mexico: PEARSON EDUCACION.

Mallo, C. (2000). Contabilidad analítica, costes, rendimientos, precios y resultados. España: Instituto de Contabilidad y auditoría de cuentas.

Mendoza, T. S. (2010). Contabilidad de costos, análisis para la toma de decisiones. (Tercera ed.). Mexico: McGrawHill Educación. 
Rendón, G. Y. (2014). Modelo de costeo basado en las actividades para instalaciones hoteleras del destino turistico Jardines del Rey. Tesis Doctoral: Universidad de Camagüey, Cuba.

Robles, R. C. (2012). Fundamentos de administración finaciera (Primera ed.). Mexico: RED TERCER MILENIO.

Zapata, S. P. (2007). Contabilidad de costos, herramineta para la toma de decisiones. (Primera ed.). Bogotá: McGrawHill Interamericana.

\section{Licencia Creative Commons}

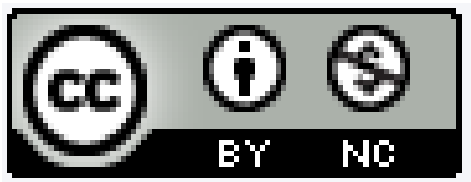

Revista Científica ECOCIENCIA está bajo una Licencia Creative Commons Atribución-NoComercial 4.0 Internacional. 